Check for updates

Cite this: Nanoscale Adv., 2019, 1, 1589

\section{Nano-MgO/AB decorated separator to suppress shuttle effect of lithium-sulfur battery $\dagger$}

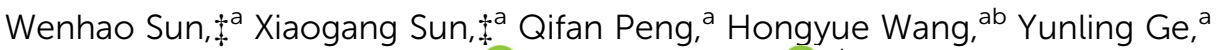 \\ Naseem Akhtar, ${ }^{a}$ Yaqin Huang (DD *a and Kai Wang (D)*b
}

Lithium-sulfur ( $\mathrm{Li}-\mathrm{S}$ ) batteries are regarded as one of the most promising energy storage systems owing to their high specific energy, low cost and eco-friendliness. However, significant capacity fading caused by the shuttle of soluble polysulfides from the cathode to the anode significantly hampers their practical application. Here, we designed a nano-MgO/acetylene black (AB) decorated functional separator to suppress the shuttle of polysulfide intermediates, which can remarkably improve the electrochemical performance of $\mathrm{Li}-\mathrm{S}$ batteries. Nano-MgO with the aid of the AB conductive network exhibits superior adsorption to polysulfides due to the synergistic effect of excellent chemisorption and improved electron conductivity. The electrochemical performance of the Li-S battery highly depends on the relative amount of nano- $\mathrm{MgO}$ and $\mathrm{AB}$ in the composite coating on the separator. $\mathrm{A}$ battery with the optimal decorated separator ( $\mathrm{MgO}-25$ separator, nano-MgO and acetylene black in the weight ratio $1: 3$ ) exhibits a high initial discharge capacity of $1238 \mathrm{~mA} \mathrm{~h} \mathrm{~g}^{-1}$ with high coulombic efficiency ( 97\%) and retains a high capacity of $875 \mathrm{~mA} \mathrm{~h} \mathrm{~g}^{-1}$ after 100 cycles at $0.2 \mathrm{C}$. This study promotes the understanding of the synergistic effect of the polysulfide adsorbent and the conductive agent on the suppression of the shuttle effect, and provides a way to design polysulfide-blocking barriers for Li-S batteries.
Received 29th December 2018 Accepted 9th February 2019

DOI: $10.1039 / c 8 n a 00420$ j

rsc.li/nanoscale-advances large volume change of the active material during the charge/ discharge process can destroy the morphology and structure of the electrode and leads to the shedding of the active material; ${ }^{7,8}$ (iii) the shuttle phenomenon of dissolved polysulfide intermediates $\left(\mathrm{Li}_{2} \mathrm{~S}_{n}, 4<n \leq 8\right)$ from the cathode to the anode results in severe self-discharge, thus causing capacity loss and the short cycle life of the batteries. ${ }^{\mathbf{9}, 10}$

Over the years, extensive efforts have been devoted to addressing the "shuttle effect" in the Li-S battery system. One of the most popular methods is to trap sulfur and polysulfides within various hosts, including carbon (porous carbon, ${ }^{\mathbf{1 1 - 1 5}}$ carbon nanotubes, ${ }^{16,17}$ graphene ${ }^{18,19}$ and elemental doped carbon $^{20,21}$ ), conductive polymers, ${ }^{22,23}$ and metal-organic frameworks. ${ }^{24,25}$ Carbon materials are the most widely employed host, however, the trapped polysulfide still shows gradual loss following repeated cycles owing to the weak interaction of the physical adsorption and the open pore structure of the carbon material. Recently, researchers have proposed that a chemisorption interaction between sulfur and some hosts seems to be more efficient at trapping and immobilizing sulfur species. Compared to traditional carbon materials, metal oxides (such as $\mathrm{TiO}_{2},{ }^{26-29} \mathrm{Al}_{2} \mathrm{O}_{3},{ }^{30,31} \mathrm{MnO}_{2},{ }^{32} \mathrm{Ti}_{4} \mathrm{O}_{7},{ }^{33}$ and $\mathrm{Mg}_{0.6} \mathrm{Ni}_{0.4} \mathrm{O}^{34,35}$ ) have excellent chemisorption ability by providing rich polar active sites for the anchoring of polysulfides. Among them, nano-MgO is considered as an especially good adsorbent for polysulfides. Previous work has also demonstrated that $\mathrm{MgO}$ as a cathode additive can improve the electrochemical performance of $\mathrm{Li}-\mathrm{S}$

\footnotetext{
${ }^{a}$ Beijing Key Laboratory of Electrochemical Process and Technology for Materials, Beijing Laboratory of Biomedical Materials, Beijing University of Chemical Technology, Beijing 100029, People's Republic of China. E-mail: huangyq@mail. buct.edu.cn

${ }^{b}$ Institute of Electrical Engineering, Chinese Academy of Sciences, Beijing 100190, People's Republic of China. E-mail: wangkai@mail.iee.ac.cn

$\dagger$ Electronic supplementary information (ESI) available. See DOI: 10.1039/c8na00420j

$\ddagger$ These authors contributed equally to this work.
} 
batteries. ${ }^{36,37}$ The hydrophilic nature of the nano-MgO nanoparticles and the presence of more electropositive $\mathrm{Mg}$ sites ensure that polysulfides can effectively be trapped on the cathode side of the separator. Moreover, $\mathrm{Mg}$ is more electropositive than the other metals, such as $\mathrm{Ti}$ and $\mathrm{Mn}$, which promotes the increased electrostatic binding force between nano-MgO and the polysulfides. ${ }^{36}$

Recently, researchers have found that inserting a polysulfide barrier (so-called interlayer) between the cathode and the separator can adsorb polysulfide species. ${ }^{38-42}$ Directly coating a functional interlayer on the cathode side of the separator to block the shuttle of polysulfides is an effective strategy. Among various interlayer materials (carbon materials, metal oxides, and metal-organic frameworks), conductive carbon materials are particularly attractive, which can also act as expanded current collectors to reduce the cell resistance and to increase sulfur utilization. Similar to the sulfur cathode host, chemisorption can effectively suppress the shuttling of polysulfides. Due to the merits of nano-MgO, it is expected that the introduction of nano-MgO to the interlayer can effectively suppress polysulfide shuttling. However, it has rarely been reported to date.

Here, we present a nano-MgO/acetylene black $(\mathrm{AB})$ decorated functional separator to inhibit the diffusion of polysulfides in $\mathrm{Li}-\mathrm{S}$ batteries, as shown in Scheme 1. Nano-MgO with the aid of the $\mathrm{AB}$ conductive agent can achieve a synergistic effect of excellent chemisorption and superior electronic conductivity, which facilitates the adsorption and transformation reaction of polysulfides. Moreover, the battery performance highly depends on the relative amount of nano-MgO and $\mathrm{AB}$ in the composite

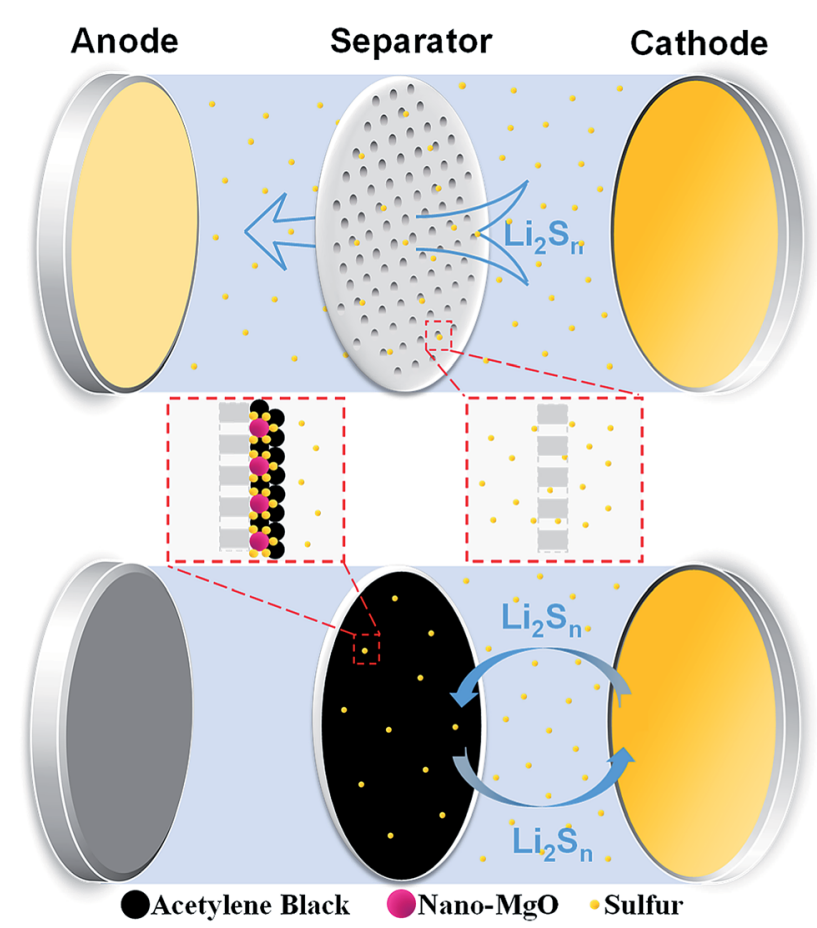

Scheme 1 Illustration of the nano-MgO decorated separator inhibiting the diffusion of polysulfides. interlayer of the separator. Insufficient nano-MgO cannot adsorb polysulfides sufficiently, resulting in poor cycling stability. On the other hand, excess nano-MgO results in an increase in the non-conductive area of battery, and polysulfides are anchored on the separator permanently without being reused, leading to low sulfur utilization. According to our experiment, a Li-S battery with the optimal separator (MgO-25 separator, $\mathrm{MgO}$ and $\mathrm{AB}$ in the weight ratio 1:3) exhibits better performance than other investigated batteries, and shows a high initial discharge capacity of $1238 \mathrm{~mA} \mathrm{~h} \mathrm{~g}^{-1}$ and a high capacity of $875 \mathrm{~mA} \mathrm{~h} \mathrm{~g}^{-1}$ after 100 cycles at $0.2 \mathrm{C}$.

\section{Experimental}

\section{Preparation of the decorated separators}

A $2 \mathrm{wt} \%$ gelatin solution was prepared by adding an appropriate amount of gelatin into deionized water. Five slurries with different weight ratios of nano-MgO and acetylene black (AB) were prepared. Nano-MgO and acetylene black (AB) were firstly mixed in various ratios by ball-milling for $5 \mathrm{~h}$ at $25{ }^{\circ} \mathrm{C}$. The content of gelatin in all slurries is the same. The weight ratios of nano-MgO and $\mathrm{AB}$ in the first three decorated separators are $1: 3,3: 3$ and $9: 3$, which are defined as the MgO-25 separator, the $\mathrm{MgO}-50$ separator and the $\mathrm{MgO}-75$ separator, respectively. The MgO-100 separator and the $\mathrm{AB}$ separator consist only of nano-MgO and $\mathrm{AB}$, respectively. The slurries were then coated on Celgard 2400 separators with an applicator, and were subsequently dried in a vacuum chamber at $60{ }^{\circ} \mathrm{C}$ for $24 \mathrm{~h}$. Lastly, the decorated separators were tablet-pressed and punched into circles with a diameter of $19 \mathrm{~mm}$. The loading mass of the decorated separators was controlled between $0.2-$ $0.4 \mathrm{mg}$.

\section{Fabrication of the cathode}

The slurry of the common sulfur cathode was prepared by mixing sublimed sulfur, $\mathrm{AB}$ and gelatin ( $2 \mathrm{wt} \%$ gelatin solution) in the weight ratio $63: 30: 7$, respectively. The slurry of the pristine $\mathrm{MgO}$ cathode was prepared by mixing nano-MgO, $\mathrm{AB}$ and gelatin in the weight ratio $20: 60: 20$, respectively. After ball-milling for $5 \mathrm{~h}$ at $25{ }^{\circ} \mathrm{C}$, the slurries were evenly coated on aluminum foil with an applicator and dried in a vacuum chamber at $60{ }^{\circ} \mathrm{C}$ for $24 \mathrm{~h}$. Finally, the cathodes were tabletpressed and punched into circles with a diameter of $12 \mathrm{~mm}$. The loading mass of sulfur in the sulfur cathode is $1.0-1.5 \mathrm{mg}$.

\section{Preparation of the $\mathrm{Li}_{2} \mathrm{~S}_{6}$-containing solution}

In a glove box under an argon atmosphere, the sublimed sulfur and $\mathrm{Li}_{2} \mathrm{~S}$ with a molar ratio of $5: 1$ were mixed in tetrahydrofuran solvent. After 24 hours of magnetic stirring, the $\mathrm{Li}_{2} \mathrm{~S}_{6}$ containing solution was prepared.

\section{Materials characterization}

The surface morphologies of the differently decorated separators and the lithium-metal after cycling were characterized by scanning electron microscopy (SEM, HITACHIS-4800) and high resolution transmission electron microscopy (HRTEM, 
HITACHIH-800). The elemental mapping check was performed using an energy dispersive spectrometer. The sulfur content of the differently decorated separator coatings was determined by thermogravimetric analysis (TGA, Hengjiu, China) in the temperature range $25-800{ }^{\circ} \mathrm{C}$ under an air atmosphere, with a heating rate of $10{ }^{\circ} \mathrm{C} \mathrm{min}^{-1}$ and an air-flow rate of 100 $\mathrm{mL} \min ^{-1}$. The crystalline phases of the nano-MgO particles and the MgO-25 separator slurry were observed by X-ray diffraction (XRD, D/max-2500/PC, Rigaku) from $10^{\circ}$ to $90^{\circ}$ with $\mathrm{Cu} \mathrm{K} \alpha$ radiation $(\lambda=0.154 \mathrm{~nm})$. UV-visual spectroscopy (UV/vis, SEC2000-DH) was used to analyze the adsorption effect of nano-MgO and $\mathrm{AB}$ on lithium polysulfide. The conductivity and wettability of the decorated separators were measured by a four-probe meter and a contact angle meter, respectively.

\section{Electrochemical measurements}

Coin-type (CR2025) cells were assembled and sealed in an argon-filled glove box, where the oxygen and water contents were less than $1 \mathrm{ppm}$. Celgard 2400 separators with/without decorated interlayers were used as the separator, and lithiummetal was used as the anode. $30 \mu \mathrm{L}$ of electrolyte was used, which consisted of 1 mol $\mathrm{L}^{-1}$ lithium bis(trifluoromethanesulfonyl)imide (LiTFSI) and a solvent of 1,3dioxolane (DOL) and 1,2-dimethoxyethane (DME) (1:1, in volume) with $0.4 \mathrm{~mol} \mathrm{~L}^{-1} \mathrm{LiNO}_{3}$ additive. The galvanostatic charge-discharge cycling of the batteries was performed in a potential range of $1.7-2.8 \mathrm{~V}\left(v s . \mathrm{Li} / \mathrm{Li}^{+}\right.$) by a LAND-CT2001A instrument. Electrochemical impedance spectroscopy (EIS) was performed on a Solartron $1280 \mathrm{Z}$ at an open-circuit voltage ranging from $100 \mathrm{MHz}$ to $10 \mathrm{kHz}$ at room temperature. Cyclic voltammetry (CV) tests were carried out with a Solartron $1280 \mathrm{Z}$ at a scan rate of $0.1 \mathrm{mV} \mathrm{s}^{-1}$.

\section{Results and discussion}

From the SEM images of $\mathrm{AB}$ (Fig. 1a) and nano-MgO (Fig. 1b), the main particle size of $\mathrm{AB}$ and nano-MgO is $\sim 50 \mathrm{~nm}$ and $\sim 100 \mathrm{~nm}$, respectively. The employed nano-MgO has obvious lattice fringes and the lattice spacing is estimated to be $0.2125 \mathrm{~nm}$ as shown in Fig. $\mathrm{S} 1, \dagger$ which is consistent with that of standard MgO (JCPDS no. 45-0946, $d=0.21056 \mathrm{~nm}$ ). Furthermore, the HRTEM image of the MgO-25 separator slurry is shown in Fig. 1c. The distribution of the nano-MgO particles in the slurry looks relatively uniform, demonstrating the sufficient blending of $\mathrm{AB}$ and nano-MgO in the composite interlayer. According to Fig. 1d, the nano-MgO and $\mathrm{MgO}-25$ separator slurries both show characteristic peaks of MgO, including (111), (200), (220), (311), and (222) in the XRD patterns. The diffraction peak of the $\mathrm{MgO}-25$ separator slurry at $2 \theta=26^{\circ}$ and is attributed to the existence of $\mathrm{AB}$. The results indicate that nano-MgO can maintain the original crystal shape well when it has been prepared as decorated separator slurries. Fig. 1e shows photographs of the six separators involved in this study, which are $\left(\mathrm{e}_{1}\right)$ the Celgard 2400 separator, $\left(\mathrm{e}_{2}\right)$ the pristine $\mathrm{MgO}$-coated $(\mathrm{MgO}-$ 100) separator, $\left(\mathrm{e}_{3}\right)$ the $\mathrm{MgO}-75$ separator, $\left(\mathrm{e}_{4}\right)$ the $\mathrm{MgO}-50$ separator, $\left(\mathrm{e}_{5}\right)$ the $\mathrm{MgO}-25$ separator and $\left(\mathrm{e}_{6}\right)$ the pristine $\mathrm{AB}$ - coated separator, respectively. The interlayer coating looks quite smooth, confirming that the functional slurries can uniformly coat on the Celgard 2400 separators. Fig. 1f and S $2 \dagger$ are cross-section SEM images of the separators, which reveal that the thickness of the commercial Celgard 2400 separator is about $20 \mu \mathrm{m}$. The nano-MgO/AB interlayer is around $5 \mu \mathrm{m}$ for all of the decorated separators except the $\mathrm{MgO}-100$ separator, for which the interlayer thickness is around $10 \mu \mathrm{m}$. Fig. 1g, S3 and $\mathrm{S} 4 \dagger$ are SEM images and EDS spectra of the MgO-25 separator, the MgO-50 separator and the MgO-75 separator, respectively, which further prove that nano-MgO is uniformly distributed in the composite interlayer of the separators. Moreover, the results indicate that the $\mathrm{AB}$ conductive network is established in the interlayer of the separators, which facilitates ion/electron transport and accelerates the transformation of the trapped polysulfides during electrochemical cycling.

Prior to making the Li-S battery with the nano-MgO decorated separator, we investigated the interaction between polysulfides and nano-MgO by directly adding nano-MgO powder into a $\mathrm{Li}_{2} \mathrm{~S}_{6}$ solution. As shown in Fig. $1 \mathrm{~h}$, the solution became clear immediately, showing that nano-MgO can adsorb polysulfides effectively. As a control experiment, the adsorption effect of pristine $\mathrm{AB}$ to polysulfides is very weak, which was further confirmed by UV-visual spectroscopy. The peak located at $330-350 \mathrm{~nm}$ (ref. 43 and 44 ) is ascribed to the existence of $\mathrm{Li}_{2} \mathrm{~S}_{6}$ and becomes weak when adding nano-MgO, showing the strong chemisorption interaction between nano-MgO and $\mathrm{Li}_{2} \mathrm{~S}_{6}$. To further confirm the interaction between polysulfides and nano-MgO, we collected XPS spectra of the $\mathrm{Mg} 2 \mathrm{p}$ region of nano-MgO before and after adsorbing $\mathrm{Li}_{2} \mathrm{~S}_{6}$ solution as shown in Fig. 1i. From the XPS spectra, the $\mathrm{Mg} 2 \mathrm{p}$ spectrum of $\mathrm{MgO} /$ $\mathrm{Li}_{2} \mathrm{~S}_{6}$ shows a $1.02 \mathrm{eV}$ shift to a lower binding energy, indicating that there is electron transfer from $\mathrm{Li}_{2} \mathrm{~S}_{6}$ to $\mathrm{Mg}$ ions. The wettability of the separator can affect the infiltration of the electrolyte and the diffusion rate of ions during the charging and discharging process. Contact angle tests were carried out to evaluate the electrolyte wettability properties on the separator. As the organic electrolyte of $\mathrm{Li}-\mathrm{S}$ batteries is seriously volatile, the contact angles of the six different separators were measured with deionized water, which possesses similar polarity to the electrolyte of $\mathrm{Li}-\mathrm{S}$ batteries. From Fig. S5, $\dagger$ the water contact angle on the pristine Celgard 2400 separator is close to $90.0^{\circ}$. However, the water contact angles on the decorated separators gradually decrease with the increase in nano-MgO content, indicating that the wettability of the separators improves with the nano-MgO functional coating.

To study the electrochemical performance of the $\mathrm{Li}-\mathrm{S}$ batteries with the $\mathrm{MgO}$ coating, the pristine $\mathrm{MgO}$ cathode (without sulfur) and the $\mathrm{Li}$ anode were first assembled into a $\mathrm{Li}-$ MgO battery. As shown in Fig. S6, $\uparrow$ there are no apparent redox peaks appearing in the cyclic voltammetry $(\mathrm{CV})$ curves of the $\mathrm{Li}-$ $\mathrm{MgO}$ battery in the working voltage range $1.7 \mathrm{~V}$ to $2.8 \mathrm{~V}$. Furthermore, Fig. S6b $\mathrm{b}^{\dagger}$ shows that the discharge specific capacity of the Li-MgO battery is only $1.6 \mathrm{~mA} \mathrm{~h} \mathrm{~g}^{-1}$ and there is no change for the 100 charge-discharge cycles, indicating that nano-MgO has no extra capacity contribution to the Li-S battery in its operating voltage range. Fig. $2 \mathrm{a}$ and Table $\mathrm{S} 1 \dagger$ show the 

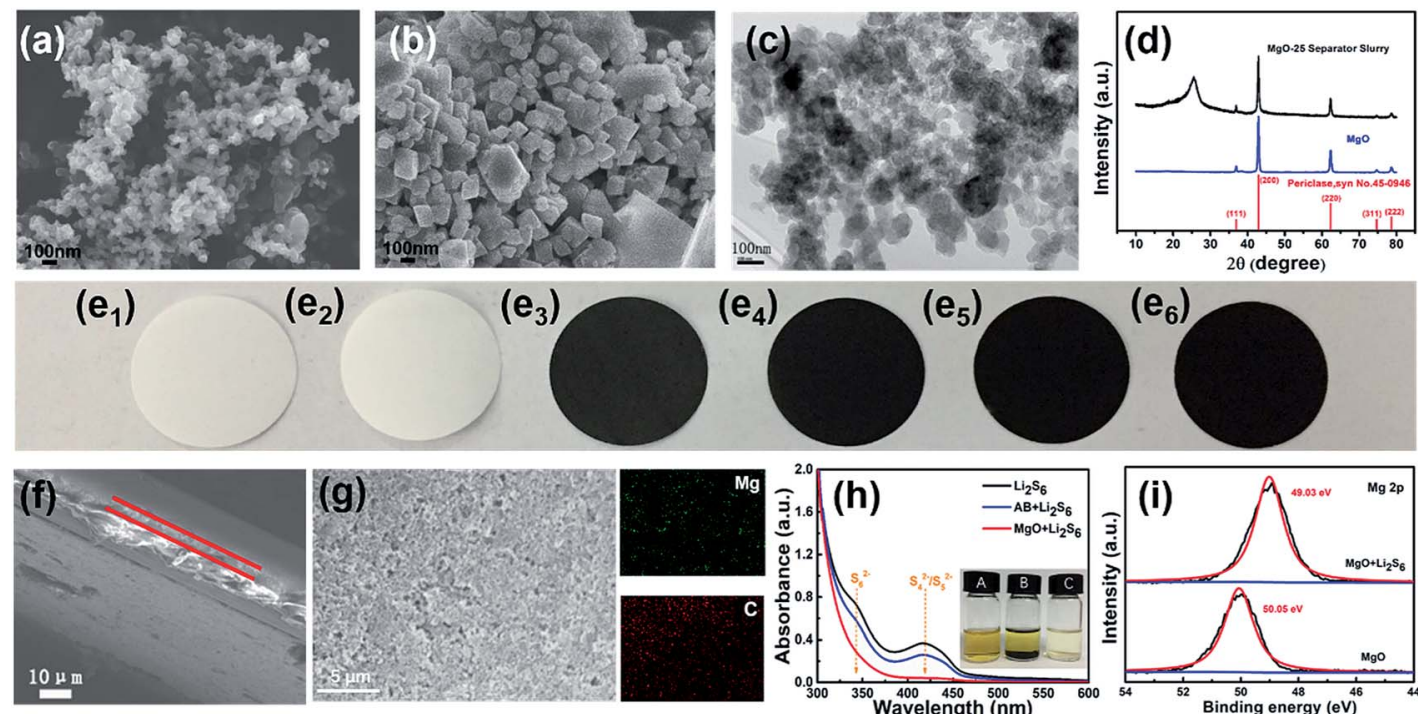

Fig. 1 SEM images of the (a) AB and (b) nano-MgO particles. (c) HRTEM image of the MgO-25 separator slurry. (d) XRD patterns of the nano-MgO particles and the $\mathrm{MgO}-25$ separator slurry. Digital images of $\left(e_{1}\right)$ the Celgard 2400 separator, $\left(e_{2}\right)$ the $M g O-100$ separator, $\left(e_{3}\right)$ the $M g O-75$ separator, $\left(\mathrm{e}_{4}\right)$ the $\mathrm{MgO}-50$ separator, $\left(\mathrm{e}_{5}\right)$ the $\mathrm{MgO}-25$ separator and $\left(\mathrm{e}_{6}\right)$ the AB-coated separator. (f) Cross-sectional SEM image of the MgO-25 separator. (g) SEM image and EDS spectra of the MgO-25 separator. (h) Digital image and UV-vis spectroscopy of the pristine and mixed $\mathrm{Li}_{2} \mathrm{~S}_{6}$ solution with nano- $\mathrm{MgO}$ and $\mathrm{AB}$ powder. (Inset (A) pristine $\mathrm{Li}_{2} \mathrm{~S}_{6}$ solution, inset (B) $\mathrm{AB}+\mathrm{Li}_{2} \mathrm{~S}_{6}$ solution, inset (C) nano-MgO $+\mathrm{Li} 2 \mathrm{~S}_{6}$ solution.) (i) $\mathrm{XPS}$ spectra of the $\mathrm{Mg} 2 \mathrm{p}$ region of nano- $\mathrm{MgO}$ before and after adsorbing $\mathrm{Li}_{2} \mathrm{~S}_{6}$ solution. (a)

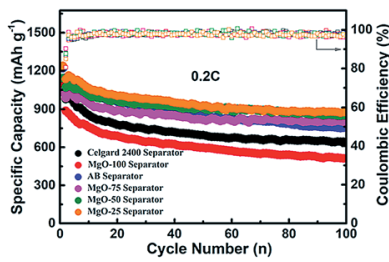

(b)

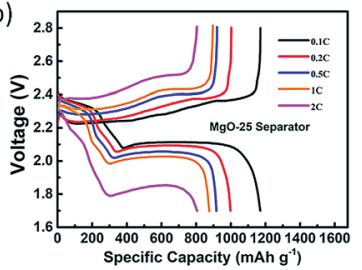

(c)

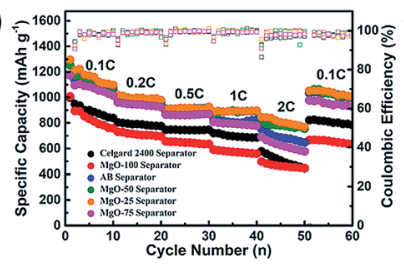

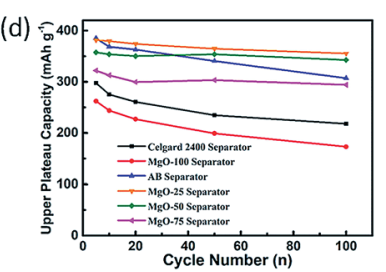

Fig. 2 (a) Cycling performance of the batteries with various separators at $0.2 \mathrm{C}$; (b) charge/discharge voltage curves at different rates for different batteries with the $\mathrm{MgO}-25$ separator; (c) rate capability of the batteries with the various separators; (d) first discharge plateau capacities of the different batteries at a rate of $0.2 \mathrm{C}$

capacity and cycling performance of the Li-S batteries with the various separators. Among the $\mathrm{Li}-\mathrm{S}$ batteries with the various separators, the battery with the $\mathrm{MgO}-25$ separator has the largest initial discharge specific capacity of $1238 \mathrm{~mA} \mathrm{~h} \mathrm{~g}^{-1}$ with a high coulombic efficiency ( $\sim 97 \%)$, and retains a specific capacity of $875 \mathrm{~mA} \mathrm{~h} \mathrm{~g}^{-1}$ after 100 charge-discharge cycles at 0.2 $\mathrm{C}$. The initial discharge specific capacity of the Li-S battery with the $\mathrm{AB}$ decorated separator is also very high, however, the capacity retention rate is only $61.82 \%$ after 100 cycles. This result demonstrates that nano-MgO exhibits a synergistic effect and improves both the capacity and the cycling performance of battery considerably. The charge-discharge curves of the Li-S batteries at various rates are shown in Fig. $2 \mathrm{~b}$ and $\mathrm{S} 7 . \dagger$ All curves display the typical characteristics of Li-S batteries with two discharge plateaus and one charge plateau, corresponding to the electrochemical redox reaction of the Li-S battery. The first discharge plateau (FDP) at about $2.35 \mathrm{~V}$ is attributed to the transformation of elemental sulfur into soluble polysulfides $\left(\mathrm{Li}_{2} \mathrm{~S}_{n}, 4 \leq n \leq 8\right)$ and the following discharge plateau at about $2.05 \mathrm{~V}$ is attributed to the transformation of the long-chain polysulfides into insoluble polysulfides $\left(\mathrm{Li}_{2} \mathrm{~S}_{2}\right.$ or $\left.\mathrm{Li}_{2} \mathrm{~S}\right)$. The charge plateaus are ascribed to the transformation of $\mathrm{Li}_{2} \mathrm{~S}_{2} / \mathrm{Li}_{2} \mathrm{~S}$ into $\mathrm{S}_{8}$. With increasing rate, the voltage gap between the discharge and charge plateaus is extended, caused by sulfur that has not reacted at high rates. The plateaus are flatter and the polarization is smaller for the Li-S battery with the MgO-25 separator than that of the batteries with other separators, suggesting a kinetically efficient reaction process. From Fig. 2c, the discharge specific capacity of the Li-S battery with the $\mathrm{MgO}-25$ separator is $1252.2 \mathrm{~mA} \mathrm{~h} \mathrm{~g}{ }^{-1}$ at $0.1 \mathrm{C}$, and is still 805.3 $\mathrm{mA} \mathrm{h} \mathrm{g}^{-1}$ at a rate of $2 \mathrm{C}$, exhibiting superior rate capability of all the batteries with decorated separators. Furthermore, it can recover $1121.9 \mathrm{~mA} \mathrm{~h} \mathrm{~g}^{-1}$ with a high capacity retention ratio of $95.9 \%$ when the discharge rate returns to 0.1 C. The ultrahigh reversibility demonstrates that the $\mathrm{MgO} / \mathrm{AB}$ composite coating on the MgO-25 separator can effectively react with the trapped polysulfides during the charge-discharge process. The first discharge plateau (FDP) analysis is a preassessment for polysulfide diffusion and the well-retained FDP capacitance manifests good reversible electrochemical performance, which also demonstrates the effective inhibition of polysulfide diffusion as the FDP relates to the formation of 
highly soluble polysulfides. ${ }^{45}$ The first discharge plateau (FDP) capacity of various batteries has been studied at a rate of $0.2 \mathrm{C}$, as shown in Fig. 2d. When employing the MgO-25 separator, the FDP capacity is $380.2 \mathrm{~mA} \mathrm{~h} \mathrm{~g}^{-1}$, which is approximately $90.7 \%$ of the theoretical capacity ( $\left.419 \mathrm{~mA} \mathrm{~h} \mathrm{~g}^{-1}\right)$. Moreover, the FDP capacity of the Li-S battery with the $\mathrm{MgO}-25$ separator retains $84.82 \%$ of the theoretical specific capacity after 100 cycles, while the $\mathrm{Li}-\mathrm{S}$ batteries with the $\mathrm{MgO}-50$ separator, the $\mathrm{AB}$ separator, the MgO-75 separator, the Celgard 2400 separator and the $\mathrm{MgO}-$ 100 separator show a capacity retention of $81.8 \%, 73.5 \%, 70.1 \%$, $52.0 \%$ and $40.9 \%$, respectively. Although the initial FDP capacity of the battery with the $\mathrm{AB}$ separator is $386.5 \mathrm{~mA} \mathrm{~h} \mathrm{~g}^{-1}$ (nearly $92.2 \%$ of the theoretical value), the capacity shows continuous decay due to the serious "shuttle effect". The above results demonstrate that the $\mathrm{MgO}-25$ separator can trap polysulfides and react with the trapped species effectively due to the synergistic effect of nano-MgO and $\mathrm{AB}$.

The charge/discharge profiles of the Li-S batteries with the various separators at a rate of $0.2 \mathrm{C}$ are presented in Fig. 3a-f. Among them, the profile of the $\mathrm{Li}-\mathrm{S}$ battery with the $\mathrm{MgO}-25$ separator exhibits the smallest potential difference $(\Delta E)$ between the discharge and charge plateaus due to the synergistic effect of strong chemisorption and the superior conducting network. However, the pristine Celgard separator and the pure $\mathrm{MgO}$ decorated separator show large $\Delta E$ values because their poor conductivity limits the secondary reaction of the adsorbed polysulfides. The pure $\mathrm{AB}$ coating cannot adsorb polysulfides effectively which also causes poor chargedischarge reversibility. The relative amount of $\mathrm{MgO}$ and $\mathrm{AB}$ influences the performance of the Li-S batteries greatly. As illustrated in Fig. $3 g_{1}-g_{3}$, the insufficient nano-MgO cannot adsorb polysulfides sufficiently, which is proposed to result in poor cycling stability. However, excess nano-MgO (such as the MgO-50 and $\mathrm{MgO}-75$ separators) causes an increase in the non-conductive area of the battery, and polysulfides will anchor on the separator permanently without being reused, leading to low sulfur utilization. In this case, excess $\mathrm{MgO}$ restricts the reaction kinetics and causes poor chargedischarge reversibility.

To further understand the synergistic effect of nano-MgO and $\mathrm{AB}$ on the reaction kinetic process of $\mathrm{Li}-\mathrm{S}$ batteries, the $\mathrm{CV}$ and EIS of the batteries were investigated. Fig. $4 \mathrm{a}-\mathrm{d}$ and $\mathrm{S} 8 \dagger$ show the first four $\mathrm{CV}$ cycles of the $\mathrm{Li}-\mathrm{S}$ batteries with the various separators at $0.1 \mathrm{mV} \mathrm{s}^{-1}$. The reduction peaks at $\sim 2.35 \mathrm{~V}$ and $\sim 1.94 \mathrm{~V}$ are assigned to the transformation of sulfur to soluble polysulfides $\left(\mathrm{Li}_{2} \mathrm{~S}_{n}, 4 \leq n \leq 8\right)$ and the long-chain polysulfides to insoluble $\mathrm{Li}_{2} \mathrm{~S}_{2} / \mathrm{Li}_{2} \mathrm{~S}$, respectively. In the subsequent anodic scan, the main peak at $\sim 2.44 \mathrm{~V}$ relates to the transformation of $\mathrm{Li}_{2} \mathrm{~S}$ to high-order polysulfides and sulfur. For all the assembled $\mathrm{Li}-\mathrm{S}$ batteries, the reduction peak shifts right
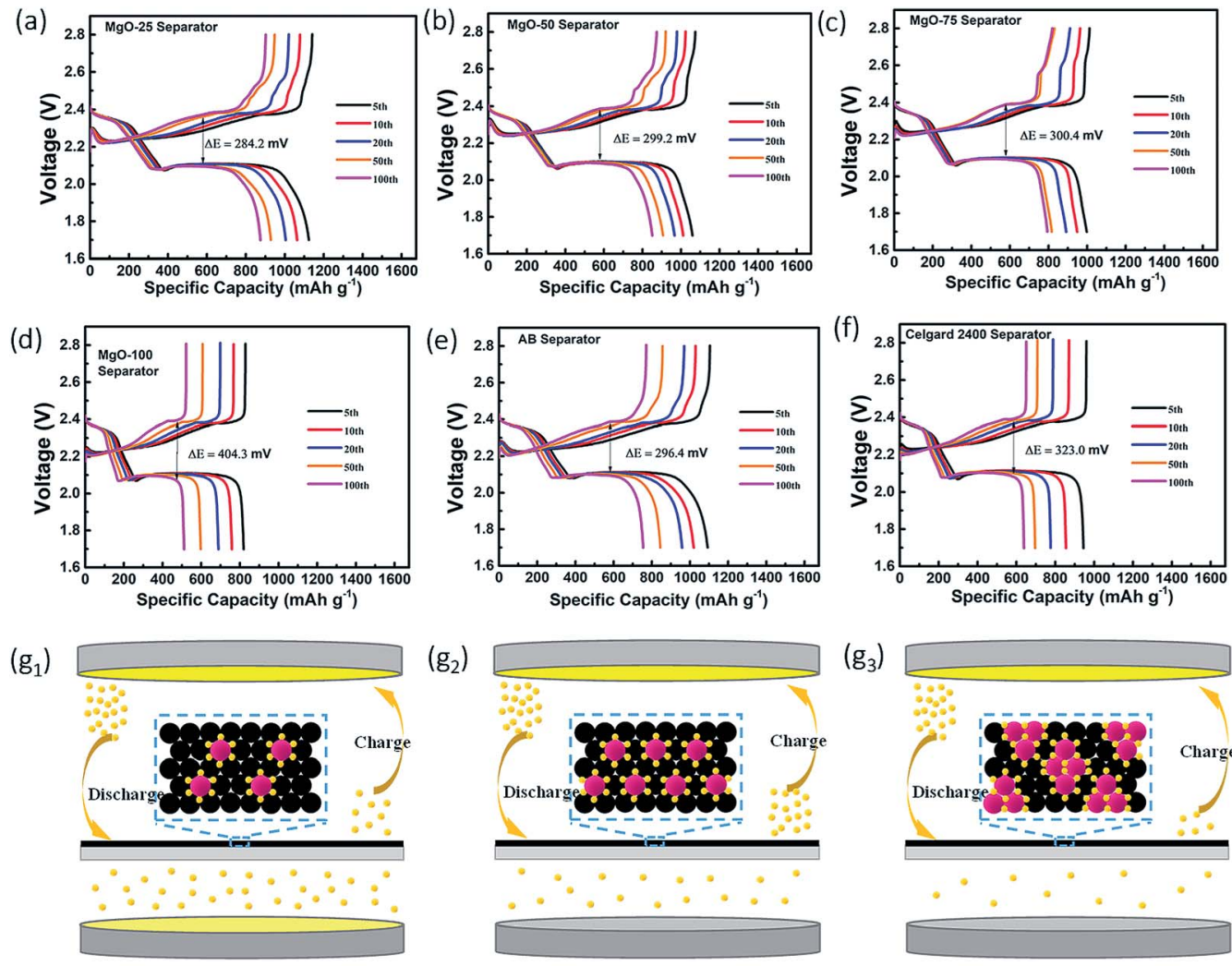

$\left(g_{2}\right)$

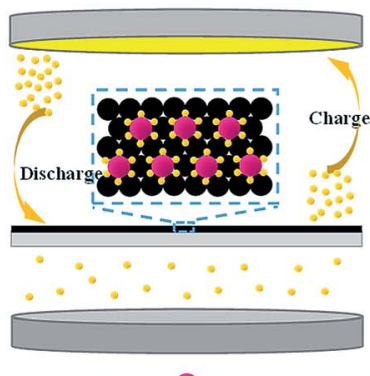

Acetylene Black ONano-MgO Sulfur

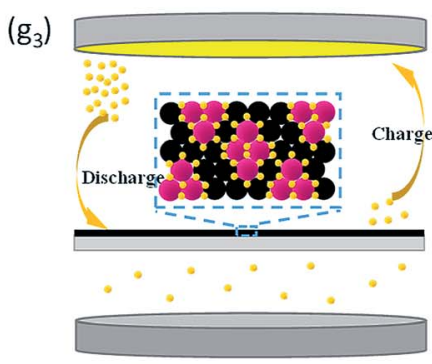

Fig. 3 Charge/discharge profiles at $0.2 \mathrm{C}$ of the $\mathrm{Li}-\mathrm{S}$ batteries with (a) the $\mathrm{MgO}-25$ separator, (b) the $\mathrm{MgO}-50$ separator, (c) the $\mathrm{MgO}-75$ separator, (d) the MgO-100 separator, (e) the AB separator and (f) the Celgard 2400 separator, respectively. Illustrations of the polysulfidestrapped/reactivated mechanisms of the differently decorated separators with $\left(g_{1}\right)$ insufficient, $\left(g_{2}\right)$ moderate and $\left(g_{3}\right)$ excess nano-MgO. 

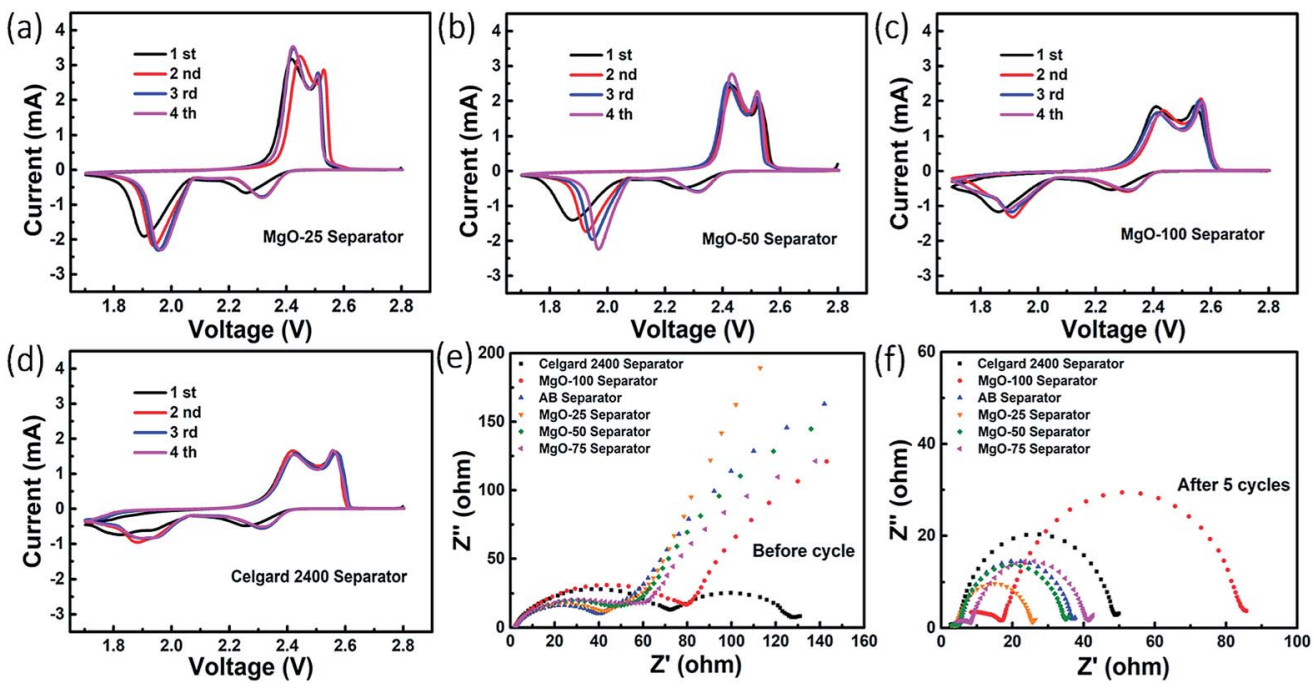

Fig. $4 \mathrm{CV}$ curves of the batteries with (a) the MgO-25 separator, (b) the MgO-50 separator, (c) the MgO-100 separator and (d) the Celgard 2400 separator. EIS spectra of the batteries with various separators (e) before cycling and (f) after 5 cycles.

gradually with increasing CV cycles, caused by irreversible diffusion of sulfur into the electrolyte and the corresponding electrode polarization. The CV curves of the Li-S battery with MgO-25 separator gradually stabilise as the CV cycles increase due to uniform secondary distribution of the sulfur on the cathode surface, suggesting excellent cycling stability and reversibility. In the fourth cycle, the voltage gaps $(\Delta E)$ between the oxidation and reduction peaks of the Li-S batteries with the $\mathrm{AB}$ separator, the $\mathrm{MgO}-25$ separator, the $\mathrm{MgO}-50$ separator, the MgO-75 separator, the MgO-100 separator and the Celgard 2400 separator are $589.6 \mathrm{mV}, 544.43 \mathrm{mV}, 552.98 \mathrm{mV}, 638.12 \mathrm{mV}$, $674.13 \mathrm{mV}$, and $662.23 \mathrm{mV}$, respectively. This indicates that the battery with the $\mathrm{MgO}-25$ separator possesses the smallest $\Delta E$. In contrast to the other Li-S batteries, the $\mathrm{CV}$ curves of the battery with the MgO-25 separator exhibit sharper peaks, demonstrating less polarization. Thus, only the appropriate amount of $\mathrm{MgO}$ and $\mathrm{AB}$ exhibits the synergistic effect of excellent chemisorption and superior electron conductivity.

Fig. 4e shows Nyquist plots of the Li-S batteries with various decorated separators. The semicircle in the high-frequency region represents the charge transfer resistance $\left(R_{\mathrm{ct}}\right)$ at the interface between the cathode and the electrolyte, while the inclined line in the low-frequency region corresponds to the Warburg impedance produced from the diffusion of electrolyte ions in the electrode. The Nyquist plots of the Li-S batteries with the $\mathrm{AB}$ separator and the $\mathrm{MgO}-25$ separator almost overlap in the high frequency region and show the smallest $R_{\mathrm{ct}}$, which confirms their excellent electronic conductivity. However, a second semicircle appears in the intermediate-frequency region for the battery with the pristine Celgard 2400 separator, which is attributed to battery self-discharge during the static process, indicating increased resistance. For all the tested batteries, another semicircle appears in the medium-to-high frequency region after 5 cycles at a rate of $0.5 \mathrm{C}$, representing the deposition resistance $\left(R_{\mathrm{d}}\right)$ of $\mathrm{Li}_{2} \mathrm{~S} / \mathrm{Li}_{2} \mathrm{~S}_{2}$ (ref. 46) as shown in Fig. $4 \mathrm{f}$. It is worth noting that the $R_{\mathrm{ct}}$ and $R_{\mathrm{d}}$ values of the battery with the MgO-25 separator are the smallest among all of the asprepared batteries that are presented in detail in Table S2. $\dagger$ The significant reduction in resistance further confirms the synergistic effect of nano-MgO and $\mathrm{AB}$, where the $\mathrm{AB}$ conductive network facilitates electron conduction during the reactivation of the adsorbed polysulfides.

Ex situ observation of the separator and the Li-metal anode before and after charge-discharge cycling was performed to validate the polysulfide adsorption effect. The surface morphology of the MgO-25 separator (Fig. 5a and b) and the MgO-100 separator (Fig. $5 \mathrm{c}$ and d) before and after cycling were checked by SEM. The nano-MgO particles uniformly distribute on the surface of the separators before cycling. After 100 cycles at a rate of $0.2 \mathrm{C}$, polysulfides deposit on the surface of the $\mathrm{MgO}-$ 25 separator due to the strong interaction between $\mathrm{MgO}$ and the polysulfides. In contrast, a thick polysulfide aggregation layer on the surface of the MgO-100 separator is observed after cycling, which is due to excess nano-MgO adsorbing many more polysulfides which cannot transform into sulfur sufficiently without the aid of the AB conducting network. Fig. 5e-j show photographs of the various separators after 5 charge-discharge cycles at a rate of $0.5 \mathrm{C}$. The surface of the separators with nano$\mathrm{MgO}$ in Fig. 5e-h are dark orange, implying more polysulfides are adsorbed on the surface. However, the color of the $\mathrm{AB}$ decorated separator and the pristine Celgard 2400 separator appears light orange, demonstrating a weak adsorption to polysulfides (Fig. $5 \mathrm{i}$ and j). Surface photographs (Fig. $5 \mathrm{e}_{1}-\mathrm{j}_{1}$ ) and SEM images (Fig. $5 \mathrm{e}_{2}-\mathrm{j}_{2}$ ) of the Li-metal anode of the batteries are also shown. From Fig. $5 \mathrm{e}_{1}$ and $\mathrm{e}_{2}$, the surface of the lithium anode in the battery with the MgO-25 separator is the smoothest, meaning that the MgO-25 separator can effectively prevent polysulfides from depositing on the Li-metal anode. Thus, the ex situ characterization results further confirm that the MgO-25 decorated separator can effectively adsorb polysulfides and suppress the shuttle effect of Li-S batteries. 

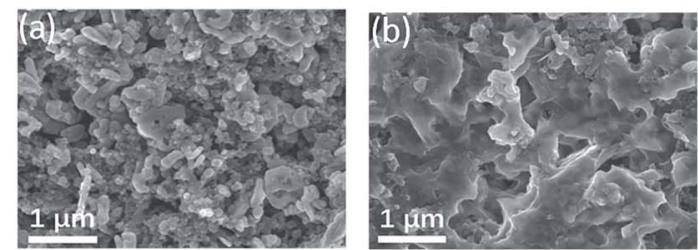

(e)

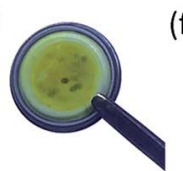

$\left(\mathrm{e}_{1}\right)$
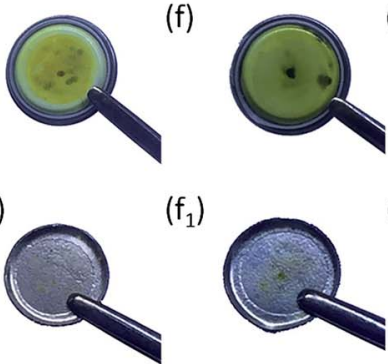

(g)
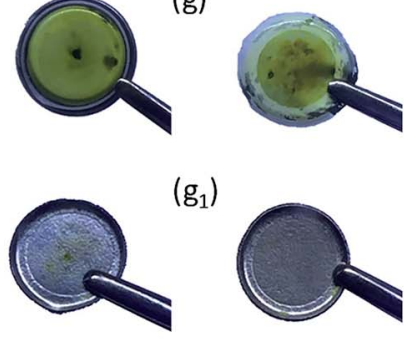

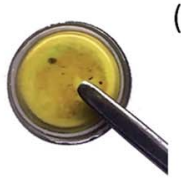

(h)

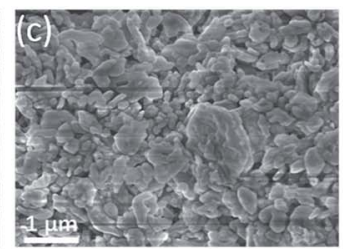

(i)

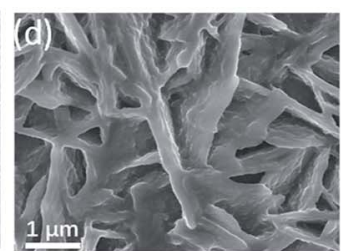

(j)

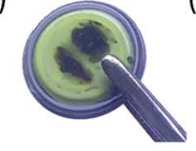

$\left(i_{1}\right)$

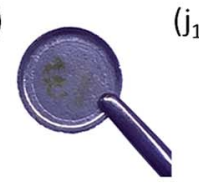

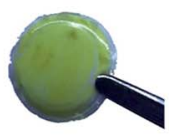

$\left(j_{1}\right)$
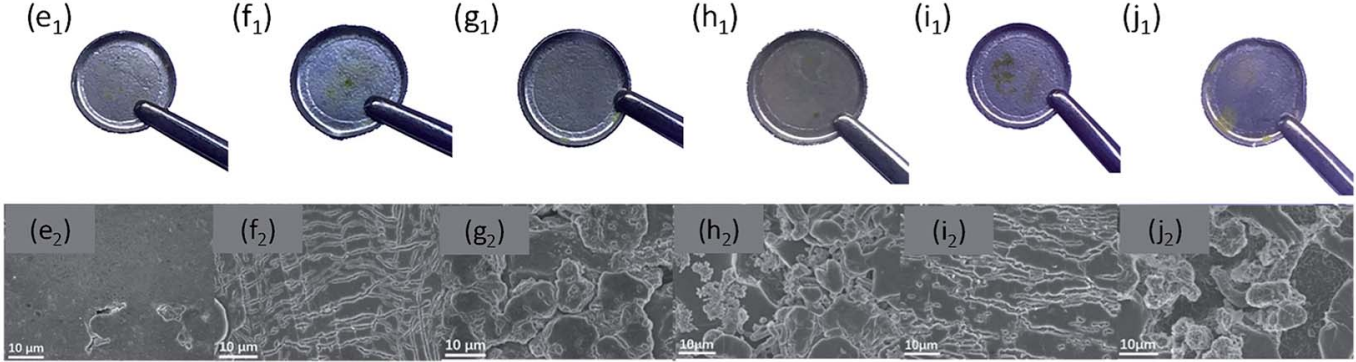

Fig. 5 Surface morphology of the $\mathrm{MgO}-25$ separator and the $\mathrm{MgO}-100$ separator ( $\mathrm{a}$ and $\mathrm{c}$ ) before and (b and d) after cycling, respectively. Photographs of the various separators after 5 cycles: (e) MgO-25 separator, (f) MgO-50 separator, (g) MgO-75 separator, (h) MgO-100 separator (i) AB separator, and (j) Celgard 2400 separator. Photographs and corresponding SEM images of the Li-metal anodes of the batteries with various separators: $\left(\mathrm{e}_{1}\right.$ and $\left.\mathrm{e}_{2}\right) \mathrm{MgO}-25$ separator, $\left(\mathrm{f}_{1}\right.$ and $\left.\mathrm{f}_{2}\right) \mathrm{MgO}-50$ separator, $\left(\mathrm{g}_{1}\right.$ and $\left.\mathrm{g}_{2}\right) \mathrm{MgO}-75$ separator, $\left(\mathrm{h}_{1}\right.$ and $\left.\mathrm{h}_{2}\right) \mathrm{MgO}-100$ separator, $\left(\mathrm{i}_{1}\right.$ and $\left.\mathrm{i}_{2}\right)$ $A B$ separator, and $\left(j_{1}\right.$ and $\left.j_{2}\right)$ Celgard 2400 separator.

\section{Conclusions}

In summary, a nano-MgO/AB decorated separator is developed to suppress the shuttle of polysulfide intermediates for the first time. Nano-MgO with the aid of an $\mathrm{AB}$ conductive agent can achieve a synergistic effect of excellent chemisorption and superior electronic conductivity, which can adsorb polysulfides effectively and accelerate the reaction kinetics for the transformation of polysulfides into sulfur. Moreover, it is found that the battery performance highly depends on the relative amount of nano-MgO and $\mathrm{AB}$ in the composite interlayer of the separator. Insufficient nano-MgO cannot adsorb polysulfides sufficiently, resulting in poor cycling stability. On the other hand, excess nano-MgO results in an increase in the non-conductive area of the battery, and polysulfides would be anchored on the separator permanently without being reused, leading to low sulfur utilization. According to our experiments, the $\mathrm{Li}-\mathrm{S}$ battery with the optimal separator ( $\mathrm{MgO}-25$ separator, $\mathrm{MgO}$ and $\mathrm{AB}$ in the weight ratio $1: 3$ ) exhibits a high initial discharge capacity of $1238 \mathrm{~mA} \mathrm{~h} \mathrm{~g}^{-1}$ with high coulombic efficiency (97\%) and retains a high capacity of $875 \mathrm{~mA} \mathrm{~h} \mathrm{~g}^{-1}$ after 100 cycles at $0.2 \mathrm{C}$. This study promotes the understanding of the synergistic effect of the polysulfide chemical adsorbent and the conductive agent on the suppression of the "shuttle effect" of Li-S batteries, which is also helpful to guide the design of high-performance functional separators for practical high-energy-density $\mathrm{Li}-\mathrm{S}$ batteries.

\section{Conflicts of interest}

There are no conflicts to declare.

\section{Acknowledgements}

The authors greatly acknowledge the National Natural Science Foundation of China (Grant No. 51777200, 51822706 and 51672020) for financial support.

\section{Notes and references}

1 L. Qie and A. Manthiram, A facile layer-by-layer approach for high-areal-capacity sulfur cathodes, Adv. Mater., 2015, 27(10), 1694-1700.

2 X. Liang, C. Hart, Q. Pang, A. Garsuch, T. Weiss and L. F. Nazar, A highly efficient polysulfide mediator for lithium-sulfur batteries, Nat. Commun., 2015, 6, 5682.

3 N. Li, Z. Weng, Y. Wang, F. Li, H.-M. Cheng and H. Zhou, An aqueous dissolved polysulfide cathode for lithium-sulfur batteries, Energy Environ. Sci., 2014, 7(10), 3307-3312.

4 Y. X. Yin, S. Xin, Y. G. Guo and L. J. Wan, Lithium-sulfur batteries: electrochemistry, materials, and prospects, Angew. Chem., Int. Ed. Engl., 2013, 52(50), 13186-13200.

5 A. Manthiram, S. H. Chung and C. Zu, Lithium-sulfur batteries: progress and prospects, Adv. Mater., 2015, 27(12), 1980-2006. 
6 X. Gu, Y. Wang, C. Lai, J. Qiu, S. Li, Y. Hou, W. Martens, N. Mahmood and S. Zhang, Microporous bamboo biochar for lithium-sulfur batteries, Nano Res., 2014, 8(1), 129-139.

7 J. Sun, Y. Sun, M. Pasta, G. Zhou, Y. Li, W. Liu, F. Xiong and Y. Cui, Entrapment of Polysulfides by a Black-PhosphorusModified Separator for Lithium-Sulfur Batteries, $A d v$. Mater., 2016, 28(44), 9797-9803.

8 B. H. Jeon, J. H. Yeon, K. M. Kim and I. J. Chuang, Preparation and electrochemical properties of lithiumsulfur polymer batteries, J. Power Sources, 2002, 109, 89-97.

9 H. Lin, L. Yang, X. Jiang, G. Li, T. Zhang, Q. Yao, G. W. Zheng and J. Y. Lee, Electrocatalysis of polysulfide conversion by sulfur-deficient MoS2 nanoflakes for lithium-sulfur batteries, Energy Environ. Sci., 2017, 10(6), 1476-1486.

10 Y. V. Mikhaylik and J. R. Akridge, Polysulfide Shuttle Study in the Li/S Battery System, J. Electrochem. Soc., 2004, 151, A1969-A1976.

11 N. Jayaprakash, J. Shen, S. S. Moganty, A. Corona and L. A. Archer, Porous hollow carbon@sulfur composites for high-power lithium-sulfur batteries, Angew. Chem., Int. Ed. Engl., 2011, 50(26), 5904-5908.

12 X. Geng, M. Rao, X. Li and W. Li, Highly dispersed sulfur in multi-walled carbon nanotubes for lithium/sulfur battery, $J$. Solid State Electrochem., 2012, 17(4), 987-992.

13 G. He, S. Evers, X. Liang, M. Cuisinier, A. Garsuch and L. F. Nazar, Tailoring Porosity in Carbon Nanospheres for Lithium-Sulfur Battery Cathodes, ACS Nano, 2013, 7, 10920-10930.

14 M. Gao, C. Su, M. He, T. Glossmann, A. Hintennach, Z. Feng, Y. Huang and Z. Zhang, A high performance lithium-sulfur battery enabled by a fish-scale porous carbon/sulfur composite and symmetric fluorinated diethoxyethane electrolyte, J. Mater. Chem. A, 2017, 5(14), 6725-6733.

15 S. Zhao, C. Li, W. Wang, H. Zhang, M. Gao, X. Xiong, A. Wang, K. Yuan, Y. Huang and F. Wang, A novel porous nanocomposite of sulfur/carbon obtained from fish scales for lithium-sulfur batteries, J. Mater. Chem. A, 2013, 1(10), 3334-3339.

16 L. Yin, J. Wang, J. Yang and Y. Nuli, A novel pyrolyzed polyacrylonitrile-sulfur@MWCNT composite cathode material for high-rate rechargeable lithium/sulfur batteries, J. Mater. Chem., 2011, 21(19), 6807-6810.

17 J.-Q. Huang, Q. Zhang, S.-M. Zhang, X.-F. Liu, W. Zhu, W.-Z. Qian and F. Wei, Aligned sulfur-coated carbon nanotubes with a polyethylene glycol barrier at one end for use as a high efficiency sulfur cathode, Carbon, 2013, 58, 99-106.

18 H. Wang, Y. Yang, Y. Liang, J. T. Robinson, Y. Li, A. Jackson, Y. Cui and H. Dai, Graphene-wrapped sulfur particles as a rechargeable lithium-sulfur battery cathode material with high capacity and cycling stability, Nano Lett., 2011, 11(7), 2644-2647.

19 R. Fang, S. Zhao, S. Pei, X. Qian, P. X. Hou, H. M. Cheng, C. Liu and F. Li, Toward More Reliable Lithium-Sulfur Batteries: An All-Graphene Cathode Structure, ACS Nano, 2016, 10(9), 8676-8682.
20 J.-J. Chen, R.-M. Yuan, J.-M. Feng, Q. Zhang, J.-X. Huang, G. Fu, M.-S. Zheng, B. Ren and Q.-F. Dong, Conductive Lewis Base Matrix to Recover the Missing Link of Li2S8 during the Sulfur Redox Cycle in Li-S Battery, Chem. Mater., 2015, 27(6), 2048-2055.

$21 \mathrm{H}$. Liu, Y. Cao, F. Wang and Y. Huang, Nitrogen-doped hierarchical lamellar porous carbon synthesized from the fish scale as support material for platinum nanoparticle electrocatalyst toward the oxygen reduction reaction, $A C S$ Appl. Mater. Interfaces, 2014, 6(2), 819-825.

22 Y. Qiu, W. Li, G. Li, Y. Hou, L. Zhou, H. Li, M. Liu, F. Ye, X. Yang and Y. Zhang, Polyaniline-modified cetyltrimethylammonium bromide-graphene oxide-sulfur nanocomposites with enhanced performance for lithiumsulfur batteries, Nano Res., 2014, 7(9), 1355-1363.

23 L. Qiu, S. Zhang, L. Zhang, M. Sun and W. Wang, Preparation and enhanced electrochemical properties of nano-sulfur/ poly(pyrrole-co-aniline) cathode material for lithium/sulfur batteries, Electrochim. Acta, 2010, 55(15), 4632-4636.

24 Z. Wang, X. Li, Y. Cui, Y. Yang, H. Pan, Z. Wang, C. Wu, B. Chen and G. Qian, A Metal-Organic Framework with Open Metal Sites for Enhanced Confinement of Sulfur and Lithium-Sulfur Battery of Long Cycling Life, Cryst. Growth Des., 2013, 13(11), 5116-5120.

25 T. Chen, B. Cheng, G. Zhu, R. Chen, Y. Hu, L. Ma, H. Lv, Y. Wang, J. Liang, Z. Tie, Z. Jin and J. Liu, Highly Efficient Retention of Polysulfides in "Sea Urchin"-Like Carbon Nanotube/Nanopolyhedra Superstructures as Cathode Material for Ultralong-Life Lithium-Sulfur Batteries, Nano Lett., 2017, 17(1), 437-444.

26 Z. Wei Seh, W. Li, J. J. Cha, G. Zheng, Y. Yang, M. T. McDowell, P. C. Hsu and Y. Cui, Sulphur-TiO2 yolkshell nanoarchitecture with internal void space for longcycle lithium-sulphur batteries, Nat. Commun., 2013, 4, 1331. 27 X. Z. Ma, B. Jin, H. Y. Wang, J. Z. Hou, X. B. Zhong, H. H. Wang and P. M. Xin, S-TiO2 composite cathode materials for lithium/sulfur batteries, J. Electroanal. Chem., 2015, 736, 127-131.

28 Q. Li, Z. Zhang, K. Zhang, L. Xu, J. Fang, Y. Lai and J. Li, Synthesis and electrochemical performance of $\mathrm{TiO} 2-$ sulfurcomposite cathode materials for lithium-sulfur batteries, J. Solid State Electrochem., 2013, 17(11), 2959-2965.

29 H. Shao, W. Wang, H. Zhang, A. Wang, X. Chen and Y. Huang, Nano-TiO2 decorated carbon coating on the separator to physically and chemically suppress the shuttle effect for lithium-sulfur battery, J. Power Sources, 2018, 378, 537-545.

30 K. Dong, S. Wang, H. Zhang and J. Wu, Preparation and electrochemical performance of sulfur-alumina cathode material for lithium-sulfur batteries, Mater. Res. Bull, 2013, 48(6), 2079-2083.

31 M. Yu, W. Yuan, C. Li, J.-D. Hong and G. Shi, Performance enhancement of a graphene-sulfur composite as a lithium-sulfur battery electrode by coating with an ultrathin $\mathrm{Al} 2 \mathrm{O} 3$ film via atomic layer deposition, J. Mater. Chem. A, 2014, 2(20), 7360-7366. 
32 X. Song, G. Chen, S. Wang, Y. Huang, Z. Jiang, L. X. Ding and H. Wang, Self-Assembled Close-Packed MnO2 Nanoparticles Anchored on a Polyethylene Separator for Lithium-Sulfur Batteries, ACS Appl. Mater. Interfaces, 2018, 10(31), 2627426282.

33 X. Tao, J. Wang, Z. Ying, Q. Cai, G. Zheng, Y. Gan, H. Huang, Y. Xia, C. Liang, W. Zhang and Y. Cui, Strong sulfur binding with conducting Magneli-phase $\mathrm{Ti}(\mathrm{n}) \mathrm{O} 2(\mathrm{n}-1)$ nanomaterials for improving lithium-sulfur batteries, Nano Lett., 2014, 14(9), 5288-5294.

34 Y. Zhang, Y. Zhao, A. Yermukhambetova, Z. Bakenov and P. Chen, Ternary sulfur/polyacrylonitrile/Mg0.6Ni0.4O composite cathodes for high performance lithium/sulfur batteries, J. Mater. Chem. A, 2013, 1(2), 295-301.

35 H. Tang, S. Yao, M. Jing, X. Wu, J. Hou, X. Qian, D. Rao, X. Shen, X. Xi and K. Xiao, Mg0.6Ni0.4O hollow nanofibers prepared by electrospinning as additive for improving electrochemical performance of lithium-sulfur batteries, $J$. Alloys Compd., 2015, 650, 351-356.

36 R. Ponraj, A. G. Kannan, J. H. Ahn and D. W. Kim, Improvement of Cycling Performance of Lithium-Sulfur Batteries by Using Magnesium Oxide as a Functional Additive for Trapping Lithium Polysulfide, ACS Appl. Mater. Interfaces, 2016, 8(6), 4000-4006.

37 M. Xiang, H. Wu, H. Liu, J. Huang, Y. Zheng, L. Yang, P. Jing, Y. Zhang, S. Dou and H. Liu, A Flexible 3D Multifunctional MgO-Decorated Carbon Foam@CNTs Hybrid as SelfSupported Cathode for High-Performance Lithium-Sulfur Batteries, Adv. Funct. Mater., 2017, 27(37), 1702573.

38 H. Shao, B. Huang, N. Liu, W. Wang, H. Zhang, A. Wang, F. Wang and Y. Huang, Modified separators coated with a $\mathrm{Ca}(\mathrm{OH}) 2$-carbon framework derived from crab shells for lithium-sulfur batteries, J. Mater. Chem. A, 2016, 4(42), 16627-16634.
39 H. Shao, F. Ai, W. Wang, H. Zhang, A. Wang, W. Feng and Y. Huang, Crab shell-derived nitrogen-doped micro-/ mesoporous carbon as an effective separator coating for high energy lithium-sulfur batteries, J. Mater. Chem. A, 2017, 5(37), 19892-19900.

40 N. Liu, B. Huang, W. Wang, H. Shao, C. Li, H. Zhang, A. Wang, K. Yuan and Y. Huang, Modified Separator Using Thin Carbon Layer Obtained from Its Cathode for Advanced Lithium Sulfur Batteries, ACS Appl. Mater. Interfaces, 2016, 8(25), 16101-16107.

41 Y. Guan, A. Wang, S. Liu, Q. Li, W. Wang and Y. Huang, Protecting lithium anode with LiNO3/Al2O3/PVDF-coated separator for lithium-sulfur batteries, J. Alloys Compd., 2018, 765, 544-550.

42 Q. Peng, F. Yu, W. Wang, A. Wang, F. Wang and Y. Huang, Ultralight polyethylenimine/porous carbon modified separator as an effective polysulfide-blocking barrier for lithium-sulfur battery, Electrochim. Acta, 2019, 299, 749-755.

43 Q. Zou and Y. C. Lu, Solvent-Dictated Lithium Sulfur Redox Reactions: An Operando UV-vis Spectroscopic Study, J. Phys. Chem. Lett., 2016, 7(8), 1518-1525.

44 C. Barchasz, F. Molton, C. Duboc, J. C. Lepretre, S. Patoux and F. Alloin, Lithium/sulfur cell discharge mechanism: an original approach for intermediate species identification, Anal. Chem., 2012, 84(9), 3973-3980.

$45 \mathrm{~S}$. H. Chung and A. Manthiram, A polyethylene glycolsupported microporous carbon coating as a polysulfide trap for utilizing pure sulfur cathodes in lithium-sulfur batteries, Adv. Mater., 2014, 26(43), 7352-7357.

46 L. Yuan, X. Qiu, L. Chen and W. Zhu, New insight into the discharge process of sulfur cathode by electrochemical impedance spectroscopy, J. Power Sources, 2009, 189(1), 127-132. 